Abanico Agroforestal. Enero-Diciembre 2020; 2:1-11. http://dx.doi.org/10.37114/abaagrof/2020.3 Artículo Original. Recibido: 06/01/2020. Aceptado: 15/04/2020. Publicado: 25/04/2020.

\title{
Calidad de la leche en los concursos de la vaca lechera en el sistema de producción familiar
}

\author{
Milk quality in the contest of the dairy cow in family system producers
Jiménez-Jiménez Randy ${ }^{1 * \underline{\mathbb{D}}}$, Rendón-Rendón María ${ }^{2} \underline{\mathrm{ID}}$, Chávez-Pérez Luis ${ }^{1} \underline{\mathrm{ID}}$, Espinosa-Ortiz Valentín ${ }^{1}$ ID

1Departamento de Economía, Administración y Desarrollo Rural, Facultad de Medicina Veterinaria y Zootecnia, Universidad Nacional Autónoma de México, Avenida Universidad 3000, C.P. 04510 Ciudad de México, México. ${ }^{2}$ Doctorado en Ciencias Agropecuarias, Universidad Autónoma Metropolitana, Unidad Xochimilco, Calzada del Hueso 1100, C.P. 04960 Ciudad de México, México. *Autor para correspondencia: Randy Jiménez-Jiménez. alexis.j2@gmail.com, mcrendon@gmail.com, luischavez_80@hotmail.com, veoee1@hotmail.com.

\section{RESUMEN}

Los concursos de la vaca lechera reconocen a la mejor vaca con base a la cantidad de leche producida, aunque, en el mercado también se considera la calidad. Con el fin de contribuir a reconocer a la mejor vaca lechera en la lechería familiar, el estudio tuvo como objetivo determinar la composición fisicoquímica y la cantidad de células somáticas (CCS) de la leche de las vacas participantes en el concurso de "La Vaca Lechera" de Maravatío, Michoacán, México. Se determinaron los principales componentes fisicoquímicos (grasa, proteína y solidos no grasos) y la CCS en la leche. Los resultados mostraron que la vaca ganadora obtuvo una producción promedio de $39 \pm 1.05$ litros/día, sin embargo, no tuvo los mejores componentes y ni la menor CCS. La mejor vaca respecto a los componentes fisicoquímicos obtuvo en promedio $29.5 \pm 1.05$ litros/día de leche, con $3.07 \%$ de proteína, $8.35 \%$ de SNF y $3.26 \%$ de grasa. La vaca que obtuvo menor CCS $(34,500 \mathrm{CS} / \mathrm{ml})$ fue la de menor producción. Se evidenció que las mejores características fisicoquímicas y de CCS en las vacas del concurso no se concentran en un solo animal; es importante que la mejor vaca, además de ser la de mayor producción debería cumplir con los mínimos requerimientos de calidad que el contexto indique.

Palabras clave: calidad de leche, grasa, lechería familiar, mejor vaca y proteína.

\section{ABSTRACT}

Dairy cow contests recognize the best cow based on the amount of milk produced, however: quality is also considered in the market. In order to contribute to recognizing the best cow in the dairy family, this study was aimed to determine the physicochemical composition and the somatic cells count (SCC) in the milk of the cows participating in the "The Dairy Cow" contest from Maravatío, Michoacán, Mexico. The main physicochemical components (fat, protein and non-fat solids) and the CCS in milk were determined. Results showed that the winning cow obtained an average production of $39 \pm 1.05$ liters/day. However, the milk did not have the best components nor the lowest SCC. The best cow regarding the physicochemical components obtained an average of $29.5 \pm 1.05$ liters/day of milk, with $3.07 \%$ protein, $8.35 \%$ solids non-fat and $3.26 \%$ fat. The cow with the lowest CCS $(34,500 \mathrm{SC} / \mathrm{ml})$ was the one with the lowest production. It was shown that the best physicochemical and SCC characteristics in the contest cows do not concentrate on a single animal. In addition, it is important to say that the best cow, apart from being the one with the highest production, should meet the minimum quality requirements according to the context.

Keywords: best cow, family dairy, fat, milk quality, protein. 


\section{INTRODUCCIÓN}

Los concursos de las vacas lecheras son realizados por instituciones gubernamentales 0 no gubernamentales, ya sean locales, estatales o nacionales; los cuales permite impulsar y fortalecer la producción de leche en la región; además de crear interés en la producción lechera entre ganaderos y en el público general que asiste. En estos eventos se invitan a los ganaderos, regularmente los más grandes y especializados en un foro, expo o feria; donde presentan a sus mejores animales para competir durante un periodo corto (regularmente durante una semana). Las vacas son ordeñadas en ese lugar, para medir su producción, y al propietario de la vaca que tenga el mayor promedio de leche producida durante ese periodo se le otorga un premio monetario. De esta manera se busca que se incremente el interés del ganadero o productor por la cría de animales de mejor genética $y$, por ende, aumente sus parámetros productivos.

La participación de los productores en estos eventos también les representa una gran herramienta de marketing. La exposición de los animales en los eventos les permite que su nombre, la familia, su establecimiento y sus animales sean admirados, vistos y reconocidos por otros. Es un espacio en el que convergen productores y donde pueden establecer relaciones comerciales para la compraventa de animales o de otros productos. Esto es mejor si alguna de sus vacas es la que tiene la mayor producción y es acreedora a los principales puestos del concurso, lo que les otorga más admiración y prestigio dentro del gremio y en la región, mejorando aún más las posibilidades de comercialización. Estos eventos son un buen canal para poder comercializar algunos de sus ejemplares a precios diferenciados.

Pese a lo anterior, no existen reportes acerca de la promoción de estos concursos para los sistemas familiares de producción de leche en México. Esto sucede porque este tipo de productores se consideran poco productivos, con vacas de pobre calidad genética y con muy baja producción; los reportes al respecto muestran promedios de producción que oscilan alrededor de 12 litros por vaca al día (Camacho-Vera et al., 2017; Chávez et al., 2018; Espinosa et al., 2011; Jiménez-Jiménez et al., 2011). Por tal motivo, la lechería familiar debe de adoptar lo que se desarrolla en otras formas especializadas de producción de leche y adquirir la genética que se desarrolla bajo otros parámetros y contextos.

Como se mencionó, en estos eventos se premia a la mejor vaca lechera con base a la cantidad de leche producida; sin embargo, la mejor vaca en el mercado o en el negocio de la leche, no es necesariamente la que produce más leche. Para muchos ganaderos las mejores vacas son aquellas que le dan un becerro al año, que sea sana, que pueda producir la mayor parte de su vida y sostener la producción durante su ciclo productivo. Asimismo, buscan que la leche contenga un mínimo de componentes fisicoquímicos e higiénicos que les cubra sus requerimientos para la elaboración de sus productos, como queso, crema, yogurt, entre otros. 
Los sólidos totales en leche y en particular el contenido de proteína y grasa, son de los principales componentes que en las últimas décadas la industria láctea ha puesto interés para mantener e incrementar su rendimiento y sus ganancias económicas. Para esto, se ha presionado y exigido al productor, primario cubrir una cantidad mínima a través de la regularización y normatividad de los estándares globalitécnicos que se han difundido en diferentes naciones. Según reportes de la Universidad de Pensilvania, si la leche tiene mayores cantidades de grasa y proteína, el valor de la leche se incrementa en noventa centavos de dólar por cada 100 libras de peso de la leche (Heinrichs et al., 2016); o una disminución de 0.5 unidades porcentuales de los sólidos totales, puede implicar una pérdida de cinco toneladas por cada millón de litros de leche procesada (Calderón et al., 2006); de ahí la importancia de tener vacas que puedan dar buen volumen de leche con la mayor cantidad de sólidos.

De la misma manera, otro de los elementos que la industria actualmente considera para calificar la leche de los productores, es la Cantidad de Células Somáticas (CCS). El contenido de estas células ayuda a conocer el estado de salud de la glándula mamaria, en lactación o la presencia de mastitis; esta última por su relación con los componentes de la leche, es un indicador de "calidad de leche". Debido a la respuesta inflamatoria durante la mastitis, se produce dentro del animal una liberación de sustancias sanguíneas hacia la leche, de las cuales algunas son enzimas que causan ruptura de la proteína y grasa láctea; las más importantes son la lipasa y la plasmina, la primera desdobla la grasa, produce un sabor rancio y disminuye la vida comercial de la leche; en tanto que la plasmina degradará la caseína y reducirá el rendimiento quesero de la leche (Hernández y Bedolla, 2008). En este sentido la CCS es un indicador importante para la industria, por que indica que entre más CCS, el animal va a presentar una menor cantidad de sólidos en la leche.

Evaluar o calificar estos componentes de la leche, no es una práctica que se promueva en los concursos en México. La mayoría de las convocatorias de estas competencias señalan como única evaluación el volumen de leche producido; no se pone atención a los sólidos de leche, mucho menos la CCS, siendo que ambos aspectos también son importantes para promocionar y tener una mejor comercialización; no solo entre productores, sino también con los industriales de lácteos. Son pocos los lugares donde se promueven y reconocen los componentes de la leche de las mejores vacas lecheras, y los reportes al respecto se dan a través de la prensa; por ejemplo, en Nicaragua, la vaca vencedora no fue la que produjo más leche, sino la que tuvo mayor contenido de grasa con menos volumen (López, 2017). En una región de Uruguay se menciona que la mejor vaca de la raza Holando obtuvo 48.4 litros de leche al día, donde ésta poseía 3.97\% de grasa y $3.09 \%$ de proteína (Samuelle, 2015). En este mismo país, los ganadores del concurso señalan que no solo se trata de cantidad de leche, también se necesitan que la leche de las vacas tengan $4 \%$ en grasa y $3.5 \%$ de proteína, porque al final, en la cantidad de sólidos es donde se ve el fruto de invertir y cuidar a los animales (Samuelle, 2019). 
Por el lado de la investigación, los estudios que se enfoquen a mostrar las características fisicoquímicas y la cantidad de CS en las vacas de concurso son casi nulos. En un estudio realizado en el concurso de la Vaca Lechera de la Expointer 2007 en Brasil, encontraron que la vaca campeona obtuvo una producción de $81.62 \mathrm{~kg}$ de leche al día, con un porcentaje de grasa de $2.23 \%$, proteína de $2.63 \%$ y obtuvo $35,000 \mathrm{CS} / \mathrm{ml}$; y la vaca que obtuvo mejores componentes fue la que obtuvo el segundo lugar con una producción de $76.78 \mathrm{~kg}$ de leche, con $2.58 \%$ de grasa y $3.23 \%$ de proteína, pero con $285,000 \mathrm{CS} / \mathrm{ml}$ (Pinto et al., 2008). Cabe resaltar que los autores concluyen, que en general las vacas del concurso presentaron elevadas producciones de leche asociada con una leche de buena calidad. Con estos referentes, se indica que no necesariamente la vaca con mayor producción, es la que tienen los mejores componentes. Es por eso, que otro factor que se tendría que evaluar en los concursos de "la Vaca Lechera" en México es la calidad fisicoquímica de la leche y la CCS.

En ese sentido, para aportar más elementos que contribuyan a reconocer a la mejor vaca lechera de una región y en particular en la lechería familiar, el presente estudio tuvo como objetivo determinar la composición fisicoquímica y la Cantidad de Células Somáticas en las vacas que participaron en el concurso de "La Vaca Lechera" de la expo-feria 2019, del municipio de Maravatío, Michoacán.

\section{MATERIAL Y MÉTODOS}

El municipio de Maravatío, Michoacán se localiza al Noreste del estado, en las coordenadas 1954' de Latitud Norte y 10027' de Longitud Oeste, a una altura de 2,020 metros sobre el nivel del mar. Limita al Norte con el estado de Guanajuato y Epitacio Huerta, al Este con Contepec y Tlalpujahua, al Sur con Senguio, Irimbo e Hidalgo, y al Oeste con Zinapécuaro (INAFED, 2010). Su clima es templado, con temperaturas que oscilan entre $14.1^{\circ} \mathrm{C}$ y $29.9^{\circ} \mathrm{C}$, y lluvias en verano con una precipitación pluvial anual de 897.7 milímetros. Maravatío tiene condiciones deseables para el desarrollo del ganado bovino, especializado en la producción de leche (Ávila, 2014).

La actividad lechera se realiza en todo el municipio, pero particularmente en las localidades del Valle de Maravatío, como: Campo Hermoso, Pomas Dolores, Santa Elena, La Colonia, El tejero y Casa Blanca (Chávez et al., 2018; Jiménez-Jiménez et al., 2011). El tipo de producción que predomina en el municipio es del tipo familiar, el $80 \%$ del inventario de bovinos de leche se desarrollan en este tipo de unidades, que se caracterizan por ser manejadas por la familia, de cinco miembros regularmente; con hatos conformados de 2 a 20 vacas, los cuales presentan de 1 a 12 vacas en producción. El ganado es principalmente de fenotipo Holstein (en menor proporción Jersey, Pardo Suizo y sus cruzas), y los rendimientos promedio por vaca por hato oscilan en 9 litros. La comercialización de la leche la realizan principalmente con los fabricantes de quesos del municipio (Jiménez et al., 2014). De tal forma que el concurso de la vaca lechera 
representa una buena oportunidad para que los productores interactúen y revivan esta actividad tradicional del municipio.

El concurso se realizó en el marco de la expo-feria de Maravatío 2019, evento conmemora el aniversario de la fundación de Maravatío, Michoacán. El concurso se llevó acabo del 22 al 27 de abril y fue organizado por el gobierno municipal para fomentar e impulsar la actividad lechera de la región. En dicho evento participaron 4 vacas de las comunidades de Dolores y Campo Hermoso, 2 vacas con fenotipo Holstein y 2 de cruza Holstein $\times$ Montbéliarde, con una edad que oscilaba de 2 a 4 años. Las vacas estuvieron en La Expo desde el 20 de abril, para que se pudieran aclimatar al entorno unos días antes de empezar el concurso. Las vacas durante el concurso se ordeñaban dos veces al día; la primera ordeña se realizaba entre 7 y 8 a.m. y la segunda entre 8 y 9 p.m. con un total de 12 ordeños durante el concurso. En cada ordeño se realizó la medición de la leche en litros, cada día se registraba en una manta visible para todo el público.

Para conocer las características fisicoquímicas y de CCS, al final de cada ordeño (mañana y tarde) se obtuvo una muestra homogenizada de $60 \mathrm{ml}$ de la leche de cada vaca, obteniendo de esta manera 12 muestras por vaca. El CCS se realizó a través del DeLaval Cell Counter (DCC), este es un equipo portátil que funciona con batería y posee un medidor óptico de células somáticas de la leche; utiliza casettes, los cuales succionan cantidades pequeñas de leche. Ya dentro del casette, la leche se mezcla con reactivos que llegan al núcleo de las células somáticas, lo cual permite su conteo, mediante un sensor de fluorescencia (Bedolla et al., 2007).

Para los componentes fisicoquímicos de la leche, se utilizó el equipo portátil MILKOSCOPE Julie C5, el cual mediante técnicas de ultrasonido y espectro de infrarrojo, tiene la capacidad de determinar diferentes componentes de la leche (grasa, proteína, lactosa, sólidos no grasos, sólidos totales, agua adicionada) (Scope Electric, 2007). Por su precisión y rapidez en la determinación de los componentes, así como por la poca preparación que requiere la muestra, este tipo de instrumentos son de los métodos de prueba que cada vez se utilizan más en las empresas y en el trabajo de campo.

La información obtenida de cada muestra fue capturada en una base de datos en Excel de Microsoft. Para el análisis de la información se utilizó estadística descriptiva, donde se presentan los datos de CCS y porcentaje de grasa, proteína y sólidos no grasos (SNF).

\section{RESULTADOS Y DISCUSIÓN}

En la tabla 1, se puede observar la producción de leche que obtuvieron las cuatro vacas. La vaca ganadora fue la que tenía cuatro años y de fenotipo Holstein-Montbéliarde, obtuvo en total 234 litros de leche durante el concurso y con un promedio de producción diaria de 39 litros de leche, superando por más de 10 litros a las restantes. 
Tabla 1. Producción de leche obtenida de las vacas participantes

\begin{tabular}{ccc}
\multicolumn{2}{c}{ durante el concurso de "la vaca lechera”, Expo-Feria Maravatío, 2019} \\
\hline Vaca & Total & Promedio \\
\hline 1 & 234 & $39 \pm 1.095$ \\
2 & 177 & $29.5 \pm 1.049$ \\
3 & 177 & $29.5 \pm 2.81$ \\
4 & 161 & $26.8 \pm 1.169$ \\
\hline
\end{tabular}

En cuanto a las características fisicoquímicas, en la tabla 2, se puede observar que la vaca con mayor porcentaje promedio de grasa fue la vaca ganadora con $3.57 \%$, pero la que obtuvo el mayor porcentaje promedio de proteína y SNF fue la vaca 3, con 3.07\% y $8.35 \%$, respectivamente. Esta vaca obtuvo el segundo lugar con una producción en el concurso de 177 litros y 29.5 litros de leche en promedio al día.

Tabla 2. Concentración promedio de grasa, proteína, SNF y CS de la leche de las vacas participantes en el concurso de la vaca lechera, Expo-feria Maravatío 2019

\begin{tabular}{ccccc}
\hline Vaca & \% Grasa & \% Proteína & \% SNF & CCS/ml \\
\hline 1 & $3.57 \pm 0.66$ & $2.87 \pm 0.17$ & $7.88 \pm 0.71$ & $87,333 \pm 3,4074$ \\
2 & $3.27 \pm 0.20$ & $2.99 \pm 0.04$ & $8.16 \pm 0.17$ & $1,438,500 \pm 21,3160$ \\
3 & $3.26 \pm 0.18$ & $3.07 \pm 0.03$ & $8.35 \pm 0.15$ & $1,006,667 \pm 12,7761$ \\
4 & $3.34 \pm 0.44$ & $2.84 \pm 0.07$ & $7.81 \pm 0.22$ & $34,500 \pm 5,244$ \\
\hline Promedio & $3.36 \pm 0.41$ & $2.94 \pm 0.13$ & $8.05 \pm 0.43$ & $641,750 \pm 624,826$ \\
Clase 1 o A & $\geq 3.1$ & $\geq 3.0$ & mínimo 8\% & $\leq 400,000$
\end{tabular}

${ }^{*}$ NMX-F-COFOCALEC-2012, (COFOCALEC, 2014)

En el conteo de células somáticas, la vaca con menor conteo fue la vaca que obtuvo el tercer lugar con $34,500 \mathrm{CS} / \mathrm{ml}$; le siguió la vaca ganadora con $87,000 \mathrm{CS} / \mathrm{ml}$, y las otras dos restantes rebasaron el millón de células somáticas por mililitro.

Los resultados mostraron que la mejor vaca lechera del certamen no necesariamente es la que tuvo los mejores componentes de la leche y la menor cantidad de células somáticas. La vaca ganadora solo superó al resto en el porcentaje de grasa y obtuvo el tercer lugar en porcentaje de proteína. En los concursos de la vaca lechera que muestran algunos resultados al respecto reportan algo similar, Pinto et al., (2008), en su estudio obtuvo que la vaca con mejores componentes en grasa y proteína, es la que obtuvo el segundo lugar con $2.78 \%$ de grasa y $3.23 \%$ de proteína. Esta situación suele ser una característica común, se conoce que cuando se produce más cantidad de leche, los componentes disminuyen por tener un mayor factor de dilución (Ávila et al., 2014; Calderón et al., 2006; Gellrich et al., 2014). Sin embargo, de acuerdo con esto, se esperaría que las vacas con menor producción obtuvieran mejores componentes; situación que en ambos casos no sucede, tanto en los reportes de Pinto et al., (2008) como en Maravatío; los últimos lugares no tuvieron la mayor cantidad de componentes. 
Lo anterior puede explicarse por las características fenotípicas de las vacas que concursaron. Es conocido que diversas razas de ganado presentan diversa capacidad para producir más o menos componentes fisicoquímicos en la leche. Al respecto, los fenotipos reportados de las vacas del concurso, solo implicaron a la raza Holstein y a su cruza con Montbéliarde. De esta última raza se reportan promedios de proteína y grasa superiores a las Holstein en promedio (Koç, 2011; Saha et al., 2018); en las razas Holstein se reportan de 3.6 a $3.7 \%$ en grasa y 3.27 a $3.3 \%$ en proteína (Ávila et al., 2014); mientras que en la raza montbéliarde oscilan entre $3.5 \%$ en grasa y $3.9 \%$ en proteína (Maciuc et al., 2010; Montbeliarde UK, 2015), y esto es similar con las cruzas de Holstein x Montbéliarde (Malchiodi et al., 2011; Saha et al., 2018).

En el concurso las vacas con mayores componentes de grasa y proteína fueron las vacas con fenotipo Holstein x Montbéliarde, es decir la vaca 1 y 3 . Es posible que la cantidad de componentes de la leche en el concurso pudo tener más influencia por los precursores genéticos de los animales, que solo el factor de dilución que da producir más leche.

En lo que respecta a la CCS, la vaca ganadora del concurso tuvo la segunda menor Cantidad de Células Somáticas. Algo similar reportó Pinto et al., (2008) de las vacas participantes en Expointer 2007, la vaca con mayor producción tampoco obtuvo la menor CCS. Se reconoce que las CS son un indicador de la presencia de mastitis, de tal forma que a medida que aumenta el CCS hay mayor predisposición de mastitis, lo cual influye en la cantidad de leche producida (Olsson, 2017), además de provocar alteraciones en los componentes fisicoquímicos de la leche y la vida útil de los derivados lácteos (Calderón et al., 2006; Heinrichs et al., 2016).

Conteos de células somáticas mayores de 500,000 células $/ \mathrm{ml}$, indican que un tercio de las glándulas se encuentran infectadas y que la pérdida de leche debido a mastitis subclínica es mayor de 10\% (Hernández y Bedolla, 2008). Al respecto, las vacas 2 y 3 del concurso presentaron conteos superiores al millón de CS/ml, es muy probable que, por el cambio de entorno (ruido, alimentación, manejo, entre otros), por estrés, hayan presentado mastitis subclínica, ya que a medida que fueron pasando los días, la CCS fue disminuyendo; es posible que la leche por el estrés en los cambios en el entorno de la vaca y por la mastitis, sufra alteraciones en las características fisicoquímicas, como lo reporta (Gellrich et al., 2014).

Cabe resaltar que la vaca que obtuvo la menor cantidad de CS no fue la de mayor producción láctea, al contrario, fue la que obtuvo la de menor producción, y por el CCS indica que no se debió a un problema de mastitis, sino a su baja capacidad genética de producción. Se reconoce que la baja CCS se asocia al genotipo racial de las vacas. En algunos estudios se ha observado que la raza Montbéliarde presenta menos CCS (Koç, 2011; Malchiodi et al., 2011), característica que le otorga la fortaleza anatómica de sus ubres. En el concurso solo dos vacas tenían este fenotipo, una de ellas fue la vaca ganadora que obtuvo menos de $100000 \mathrm{CS} / \mathrm{ml}$, y la otra fue la vaca 3, que obtuvo más 
de 1 millón de CS/ml. Esta diferencia puede indicar que hay diferentes factores que pueden afectar el CCS y que no únicamente depende la raza.

Es notorio que las características fisicoquímicas de la leche y el conteo de células somáticas de las vacas del concurso, no se concentran en un solo animal, por lo que valorar a la mejor vaca bajo estos preceptos, no parece práctico. No siempre la vaca que tiene mayores concentraciones de proteína y grasa es la que produce más leche o la que tiene menor CCS. Un solo indicador no puede ser el que determine cual vaca es la mejor, en un contexto donde varios aspectos deben ser considerados para representar el prestigio de un productor en su gremio y en el mercado de los lácteos. Quizás la mejor vaca lechera debería ser aquella que, además de ser la más alta productora de leche, debería de cumplir con los requerimientos mínimos que el contexto le indique, como se observó en el estudio de Pinto et al., (2008), cumplir los indicadores que los actores locales acuerden y las normas o estándares que se indiquen en el país.

En México existe la norma NMX-F-COFOCALEC-2012, la cual otorga las especificaciones fisicoquímicas y sanitarias de la leche cruda de vaca. Esta norma clasifica por componentes y por CCS en diferentes clases, siendo la clase 1 o A, la mejor; para la grasa representan valores $\geq 3.1 \%$, proteína $\geq 3 \%$, lactosa entre 4.1 y $4.85 \%$, SNF mínimo $8 \%$, y para CCS/ml $\leq 400,000$ (COFOCALEC, 2014). Al respecto, la vacas que más se acercaron a cumplir con estos estándares fueron la vaca 1, la ganadora que se encontró en clase 1 para \% de grasa y CCS, para proteína en Clase C y no alcanzó el mínimo para SNF; y la vaca 3 que obtuvo clase 1 para proteína y grasa, cubrió con lo mínimo de SNF y obtuvo clase 4 para CCS. Aunque ninguna alcanza a cumplir con los mejores estándares de acuerdo con la norma. Se puede observar que la vaca 3 alcanzó más estándares, además de obtener mayor cantidad de leche que la 2 y 4 , por lo que de acuerdo con la producción y componentes podría considerarse como la mejor entre las concursantes.

En este estudio, no se pudo encontrar a la vaca que cubriera todos los requisitos, tanto en cantidad como calidad de la leche, como se sugiere en las normas. El número de vacas que participaron en el concurso puede ser una limitante para poder identificar a aquella vaca que pueda ser alta productora y también cumpla con los estándares de calidad del país, y a pesar de que el municipio cuenta con tradición en la producción de leche, muy pocos productores se animan a participar en estos concursos.

Es notorio que en la leche de las vacas del concurso, existe una heterogeneidad en su volumen, componentes y salud; lo cual es sin duda reflejo de la misma diversidad que caracteriza al sistema de producción familiar a pequeña escala, donde las características productivas son reflejo del contexto geográfico y socioeconómico en el que prevalecen estos sistemas. No obstante, las características fisicoquímicas, la CCS y volumen encontrado en las vacas de este concurso, son indicativos de que en los sistemas de producción familiar, se pueden tener vacas con buenos rendimientos en volumen y en 
calidad; por lo que es importante que se promuevan políticas públicas en apoyo a este tipo de sistemas, ya que pueden proveer una materia prima y un alimento de buena calidad que cubra la insuficiencia láctea en el país.

\section{CONCLUSIONES}

Para que una vaca se considere la mejor a nivel local, regional o nacional y que vaya acorde a las necesidades del mercado de lácteos, debería considerar tanto la producción de leche como las características fisicoquímicas y la Cantidad de Células Somáticas de la leche. Aquella vaca que produzca más leche no puede ser la mejor, si no cubre al menos los estándares que se indican en cada región o país. Por tal motivo, si se quiere impulsar y fortalecer la actividad lechera en una región, sería recomendable incluir en los concursos de la vaca lechera la evaluación de los principales componentes de la leche, así como el conteo de Células Somáticas, puesto que son indicadores que van siendo cada vez más relevantes en el mercado de los lácteos, y el mercado de los pequeños productores no es la excepción.

\section{AGRADECIMIENTOS}

Se agradece al Programa de Apoyo a Proyectos de Investigación e Innovación Tecnológica PAPIIT de la UNAM (IN309317 y IN305620) por el financiamiento para realizar el trabajo y a los productores del concurso por facilitar la información.

\section{LITERATURA CITADA}

ÁVILA TS, Núñez EJF, Gutiérrez CAJ. 2014. Factores que intervienen en calidad y cantidad de leche. En Ávila TS, Gutiérrez CAJ. Producción de leche con ganado bovino. Imagen Editorial Yire. ISBN: 13 978-607-008107-1

BEDOLLA CC, Castañeda VH, Wolter W. 2007. Métodos de detección de la mastitis bovina. REDVET. Revista electrónica de Veterinaria. 8(9). ISSN: 1695-7504. http://www.veterinaria.org/revistas/redvet/n090907/090702.pdf

CALDERÓN A, García F, Martínez G. 2006. Indicadores de calidad de leches crudas en diferentes regiones de Colombia. Revista MVZ Córdoba. 11(1). ISSN: 1909-0544. https://doi.org/10.21897/rmvz.457

CAMACHO-VERA JH, Cervantes-Escoto F, Palacios-Rangél MI, Rosales-Noriega F, Vargas-Canales JM. 2017. Factores determinantes del rendimiento en unidades de producción de lechería familiar. Revista Mexicana de Ciencias Pecuarias. 8(1): 23-29. ISSN: 2448-6698. https://doi.org/10.22319/rmcp.v8i1.4313

CHÁVEZ PLM, Espinosa OVE, Jiménez JRA, Alonso PFA, Brunett PL. 2018. La sustentabilidad de la actividad lechera en comunidades campesinas de Maravatío, Michoacán: Variaciones en el corto plazo. Revista Latinoamericana de Educación y Estudios Interculturales. 2(4): 61-72. ISSN: 2448-8801

http://cresur.edu.mx/OJS/index.php/RLEEI_CRESUR/article/view/279. 
COFOCALEC. 2014. NORMA MEXICANA NMX-F-700-COFOCALEC-2012 Sistema Producto Leche - Alimento - Lácteo - Leche Cruda de Vaca - Especificaciones Fisicoquímicas, Sanitarias y Métodos de Prueba. Consejo para el Fomento de la Calidad de la Leche y sus Derivados A.C. https://www.cofocalec.org.mx/catalogo/por_tema

ESPINOSA OVE, Jiménez JRA, Gil GGI, Alonso PA, Brunett PL y García HLA. 2011. Lechería familiar. La Jornada del campo. 9. Suplemento Informativo de La Jornada. https://www.jornada.com.mx/2011/12/17/cam-lecheria.html

GELLRICH K, Meyer HHD y Wiedemann S. 2014. Composition of major proteins in cow milk differing in mean protein concentration during the first 155 days of lactation and the influence of season as well as short-term restricted feeding in early and mid-lactation. Journal of Animal Science. 59(3):97-106. ISSN: 1212-1819, 1805-9309. https://doi.org/10.17221/7289-CJAS

HEINRICHS J, Jones C y Bailey K. 2016. Milk Components: Understanding Milk Fat and Protein Variation in Your Dairy Herd. Penn State Extension. https://extension.psu.edu/milk-components-understanding-milk-fat-and-protein-variationin-your-dairy-herd

HERNÁNDEZ RJM, Bedolla CJLC. 2008. Importancia del conteo de células somáticas en la calidad de la leche. Revista Electrónica de Veterinaria. 9(9). ISSN: 1695-7504. https://www.redalyc.org/pdf/636/63617329004.pdf

INAFED. 2010. Michoacán de Ocampo-Maravatío. http://siglo.inafed.gob.mx/enciclopedia/EMM16michoacan/municipios/16050a.html

JIMÉNEZ-JIMÉNEZ R, Espinosa OVE, Alonso PFA, Hernández, LAG, González GIG, Flores JLD. 2011. Globalization Effects in Family Farms: A Case of Mexican Dairy Production. The Economic Geography of Globalization. ISBN: 978-953-307-502-0. https://doi.org/10.5772/18763

JIMÉNEZ JRA. Espinosa OVE, Soler FDM. 2014. El costo de oportunidad de la mano de obra familiar en la economía de la producción lechera de Michoacán, México. Revista de Investigación Agraria y Ambiental. 5(1):47-56. e-ISSN: 2145-6453. https://doi.org/10.22490/21456453.908

KOÇ A. 2011. A study of the reproductive performance, milk yield, milk constituents, and somatic cell count of Holstein-Friesian and Montbeliarde cows. Turkish Journal of Veterinary and Animal Sciences. 35(5):295-302. ISSN: 1303-6181. https://doi.org/10.3906/vet-1008-18

LÓPEZ RLS. 2017. Contribución en el manejo y desarrollo de actividades pecuarias en unidad de producción. Del municipio de Yali, departamento de Jinotega, en el periodo comprendido de enero a junio 2017. Engineer, Universidad Nacional Agraria. http://repositorio.una.edu.ni/3714/ 
MACIUC V, Creanga St, Schutz M, Russel M, Ujica V. 2010. Montbeliard breed in eastern romania. Lucrări Ştiinţifice, Seria Zootehnie. 54:164-169. ISSN 2067-2330. http://www.uaiasi.ro/zootehnie/Pdf/Pdf_Vol_54/V_Maciuc.pdf

MALCHIODI F, Penasa M, Tiezzi F, Bittante G. 2011. Milk Yield Traits, Somatic Cell Score, Milking Time and Age at Calving of Pure Holstein Versus Crossbred Cow. Agriculturae Conspectus Scientificus. 76(3):259-261. ISSN 1331-7768. https://hrcak.srce.hr/72048

MONTBELIARDE UK. 2015. About the Montbeliarde Breed [Website by RGPS]. Montbeliarde. http://montbeliardeuk.co.uk/about-the-breed/

OLSSON E. 2017. Success factors for profitable dairy farming [Master's Thesis, Swedish University of Agricultural Sciences]. https://stud.epsilon.slu.se/13189/

PINTO AT, Zanela MB, Ribeiro MER, Fernández VNV, Santos J de O. 2008. Correlação entre os métodos infravermelho e ultra-som na determinação da composição química do leite das vacas do concurso leiteiro da Expointer 2007. Acta Scientiae Veterinariae. 36(3): 273-276. ISSN: 1678-0345, 1679-9216. https://seer.ufrgs.br/index.php/ActaScientiaeVeterinariae/article/view/17299/10198

SAHA S, Carraro L, Bittante G, Gallo L. 2018. Body and milk quality traits of purebred Holstein and three-generation crossbred cows from Viking Red, Montbéliarde, and Holstein sires. Journal of Central European Agriculture. 19(4):760-765. ISSN: 1332-9049. https://doi.org/10.5513/JCEA01/19.4.2334

SAMUELLE J. 2015. La vaca más lechera es maragata. El Observador. https://www.elobservador.com.uy/nota/la-vaca-mas-lechera-es-maragata-2015916500

SAMUELLE J. 2019. Las mejores vacas Holando son de El Grillo y La Muesca. El Observador. https://www.elobservador.com.uy/nota/las-mejores-vacas-holando-son-deel-grillo-y-la-muesca-201951318133

SCOPE ELECTRIC. 2007. Julie C5TMAutomatic milk analyzer from Scope Electric. Scope Electric Instruments. https://scope-electric.com/milk_analyzers/Julie_C5.htm

Publica tus resultados de investigaciones en las revistas abanico. https://abanicoacademico.mx/revistasabanico 